\title{
A SIMPLICIAL COMPLEX IS UNIQUELY DETERMINED BY ITS SET OF DISCRETE MORSE FUNCTIONS
}

\author{
NICOLAS ARIEL CAPITELLI AND ELIAS GABRIEL MINIAN
}

\begin{abstract}
We prove that a connected simplicial complex is uniquely determined by its complex of discrete Morse functions. This settles a question raised by Chari and Joswig. In the 1-dimensional case, this implies that the complex of rooted forests of a connected graph $G$ completely determines $G$.
\end{abstract}

\section{INTRODUCTION}

The complex of discrete Morse functions $\mathfrak{M}(K)$ of a finite simplicial complex $K$ was introduced by Chari and Joswig in [5] to study the topology of simplicial complexes in terms of their sets of discrete deformations. $\mathfrak{M}(K)$ is the complex of all possible discrete Morse functions on $K$ and, as remarked in [5], it can be regarded as a combinatorial analogue of the space of vector fields on a differentiable manifold. Despite the potential utility of this complex, very little was known about the relationship between $K$ and $\mathfrak{M}(K)$. Chari and Joswig studied some properties of the complexes associated to graphs and simplices and computed the homotopy type of the complex associated to the 2-simplex. Their work was shortly followed by Ayala, Fernández, Quintero and Vilches, who described the structure of the pure Morse complex of a graph $G$, i.e. the subcomplex of $\mathfrak{M}(G)$ generated by the simplices of maximal dimension [1]. As pointed out in [5], the construction of $\mathfrak{M}(K)$ in the context of graphs was already implicit in the work of Kozlov [10], who studied complexes arising from directed sub-trees of a given (directed) graph. Kozlov proved shellability of the complexes associated to complete graphs and computed the homotopy type of the complexes associated to paths and cycles.

The aim of this article is to settle the connection between a simplicial complex and its complex of discrete Morse functions. We show that $K$ is completely determined by $\mathfrak{M}(K)$. Concretely, our main result is the following.

Theorem A. Let $K, L$ be finite connected simplicial complexes. If $\mathfrak{M}(K)$ is isomorphic to $\mathfrak{M}(L)$ then $K$ is isomorphic to $L$.

For the 1-dimensional case, we prove that Theorem A also holds for multigraphs.

Theorem B. Let $G, G^{\prime}$ be finite connected multigraphs. If $\mathfrak{M}(G)$ is isomorphic to $\mathfrak{M}\left(G^{\prime}\right)$ then $G$ is isomorphic to $G^{\prime}$.

We also exhibit an example which shows that the homotopy type of $\mathfrak{M}(K)$ does not determine the homotopy type of $K$.

The results in this article provide the complete answers to the foundational questions about $\mathfrak{M}(K)$ raised by Chari and Joswig in [5].

2010 Mathematics Subject Classification. 52B05, 57Q05, 57M15, 05C10.

Key words and phrases. Discrete Morse theory, discrete Morse complex, collapsibility.

Researchers of CONICET. Partially supported by grants ANPCyT PICT-2011-0812, CONICET PIP 112-201101-00746 and UBACyT 20020130100369. 


\section{The COmplex of Discrete Morse functions}

All simplicial complexes that we deal with are assumed to be finite. We write $\sigma \prec \tau$ if the simplex $\sigma$ is an immediate face of $\tau$ (i.e. a proper maximal face) and we let $V_{K}$ denote the set of vertices of a complex $K$. We denote by $\Delta^{n}$ the standard complex consisting of all the faces of an $n$-simplex, and by $\partial \Delta^{n}$ its boundary (i.e. the complex of all the proper faces of the simplex).

A discrete vector field on an abstract simplicial complex $K$ is a map $W: K \rightarrow K \cup\{0\}$ such that

$(W 1)$ if $W(\sigma) \neq 0$ then $\sigma \prec W(\sigma)$,

$(W 2)$ if $W(\sigma)=W\left(\sigma^{\prime}\right) \neq 0$ then $\sigma=\sigma^{\prime}$, and

(W3) $W^{2}=0$.

The simplices in $W^{-1}(0) \backslash W(K)$ are called critical. If $W(\sigma)=\tau$ for some $\sigma \prec \tau$ then the pair $(\sigma, \tau)$ is called a regular pair. One can easily see that every simplex in $K$ is either critical or belongs to a unique regular pair (see $[7,8]$ for more details). If $(\sigma, \tau)$ is a regular pair, we call $\sigma$ the source simplex of the pair, and write $s(\sigma, \tau)=\sigma$, and we call $\tau$ the target simplex of the pair, and write $t(\sigma, \tau)=\tau$. The index of a regular pair $(\sigma, \tau)$ is the dimension of $\sigma$. A regular pair of index $k$ will be sometimes denoted by $\left(\sigma^{k}, \tau^{k+1}\right)$. Typically, a regular pair $(\sigma, \tau)$ is depicted graphically as an arrow from $\sigma$ to $\tau$ (see Figure $1)$.
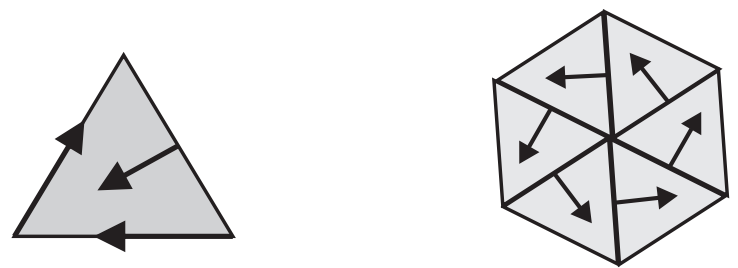

Figure 1. On the left: graphical representation of regular pairs. On the right: a cyclic vector field of index 1 .

Given two discrete vector fields $W, W^{\prime}$ over $K$ we write $W \lesssim W^{\prime}$ if every regular pair of $W$ is also a regular pair of $W^{\prime}$. Note that if $W \lesssim W^{\prime}$ and $W^{\prime} \lesssim W$ then $W=W^{\prime}$. A discrete vector field $W$ over $K$ is called cyclic of index $k$ if $(i)$ it has at least three regular pairs; (ii) every regular pair has index $k(0 \leq k \leq \operatorname{dim}(K)-1)$; and (iii) the source simplices of its regular pairs can be arrange in a sequence of $k$-simplices $\sigma_{0}, \ldots, \sigma_{r} \in$ $W^{-1}(K)$ such that $\sigma_{i} \neq \sigma_{j}$ for $i \neq j, \sigma_{0} \prec W\left(\sigma_{r}\right)$ and $\sigma_{i+1} \prec W\left(\sigma_{i}\right)$ for $0 \leq i \leq r-1$ (see Figure 1).

A combinatorial Morse function over $K$ is a map $f: K \rightarrow \mathbb{R}$ satisfying for every $\sigma \in K$

$(M 1)|\{\eta \prec \sigma \mid f(\eta) \geq f(\sigma)\}| \leq 1$ and

(M2) $|\{\tau \succ \sigma \mid f(\tau) \leq f(\sigma)\}| \leq 1$.

Here $|X|$ denotes the cardinality of the set $X$. The gradient vector field of a combinatorial Morse function $f$ is the discrete vector field $V_{f}$ over $K$ defined by

$$
V_{f}(\sigma)= \begin{cases}\tau & \text { if } \tau \succ \sigma \text { and } f(\sigma) \geq f(\tau) \\ 0 & \text { otherwise. }\end{cases}
$$

A simplex $\sigma$ is critical for $f$ if $\sigma$ is critical for $V_{f}$. Given $\sigma \prec \tau$, it is easy to see that $(\sigma, \tau)$ is a regular pair of $V_{f}$ if and only if $f(\sigma) \geq f(\tau)$. By [7, Theorem 9.3], a vector field is the gradient of a combinatorial Morse function if and only if it does not contain cyclic vector fields. 
A discrete vector field with exactly one regular pair is called a primitive vector field. Note that primitive vector fields are gradient fields. We will often identify a primitive vector field with its sole regular pair. A collection $W_{0}, \ldots, W_{r}$ of primitive vector fields is said to be compatible if there exists a gradient vector field $V$ on $K$ with $W_{i} \lesssim V$ for every $i=0, \ldots, r$. The complex of discrete Morse functions of $K$ is the simplicial complex $\mathfrak{M}(K)$ whose vertices are the primitive vector fields on $K$ and whose $r$-simplices are the discrete gradient fields with $r+1$ regular pairs. We identify in this way a gradient vector field $V$ with the set $\left\{W_{0}, \ldots, W_{r}\right\}$ of all primitive vector fields satisfying $W_{i} \lesssim V$ (i.e. the set of its regular pairs). $\mathfrak{M}(K)$ is also called the discrete Morse complex of $K$. Figure 2 shows some low-dimensional examples of discrete Morse complexes.

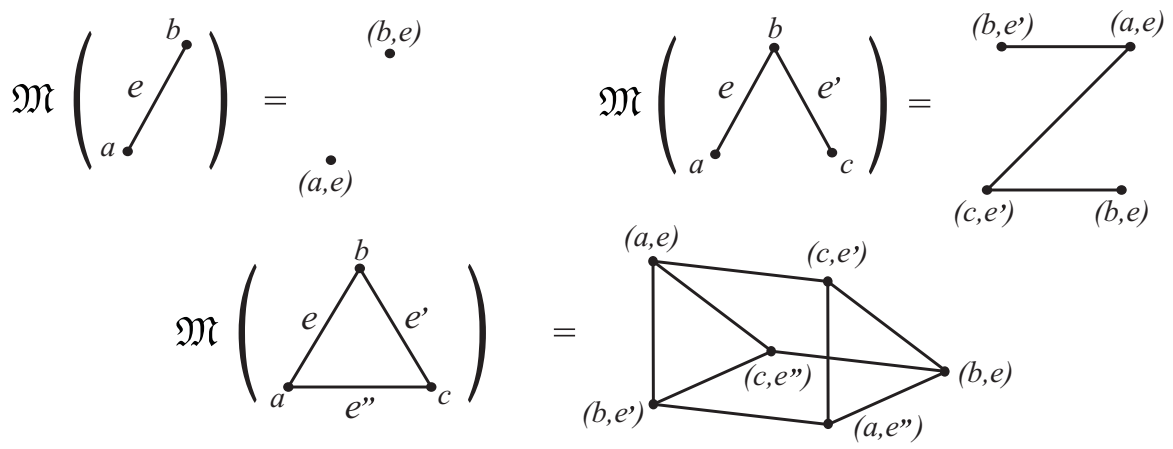

Figure 2. Examples of complexes of discrete Morse functions.

There is an alternative approach to discrete Morse theory due to Chari [4] where the deformations are encoded in terms of acyclic matchings in the Hasse diagram of the face poset of the simplicial complex. It is not hard to see that the pairing of simplices which form regular pairs of a discrete Morse function determines a matching in the Hasse diagram $\mathcal{H}_{K}$ of $K$. If the arrows in this matching are reversed, it can be easily shown that the resulting directed graph is acyclic. On the other hand, from an acyclic matching on the Hasse diagram of a simplicial complex one can build a discrete Morse function $f$ on $K$ where the regular pairs of $f$ are precisely the edges of the matching. From this viewpoint, $\mathfrak{M}(K)$ is the simplicial complex on the edges of the Hasse diagram of $K$ whose simplices are the subsets of edges which form acyclic matchings.

\section{The COMPlEXes ASSOCIATED TO GRAPHS}

The complex of discrete Morse functions has been studied almost exclusively for graphs, as the construction of $\mathfrak{M}(K)$ for a general $K$ is rather complicated (see for example $[1,5]$ ). We focus first on this case and settle the main result for 1-dimensional regular $\mathrm{CW}$ complexes (Theorem B).

Recall that a multigraph $G$ is a triple $\left(V_{G}, E_{G}, f_{G}\right)$ where $V_{G}$ is a (finite) set of vertices, $E_{G}$ is a set of edges and $f_{G}: E_{G} \rightarrow\left\{\{u, v\}: u, v \in V_{G}\right.$ and $\left.u \neq v\right\}$ is a map which assigns to each edge its boundary vertices. If $f_{G}(e)=f_{G}\left(e^{\prime}\right)$ for $e, e^{\prime} \in E_{G}$, we say that $e, e^{\prime}$ are parallel edges. For $v, v^{\prime} \in V_{G}, E_{G}\left(v, v^{\prime}\right)$ will stand for the set of parallel edges between $v$ and $v^{\prime}$. Note that, by definition, a multigraph has no loops. Simple graphs correspond to multigraphs $G$ where $f_{G}$ is injective. In this case we shall identify an edge with its boundary vertices and write $e=v w$ if $f_{G}(e)=\{v, w\}$. Note that simple graphs are precisely the 1-dimensional simplicial complexes and multigraphs are precisely the 1-dimensional regular CW-complexes (see [11] for the necessary definitions).

The complex of discrete Morse functions of a graph was first studied by Kozlov [10] under a different context. Given a directed graph $G$, Kozlov defined the simplicial complex 
$\Delta(G)$ whose vertices are the edges of $G$ and whose faces are all directed forests which are subgraphs of $G$. In [10] he studied the shellability of the complete double-directed graph on $n$ vertices (a graph having exactly one edge in each direction between any pair of vertices) and computed the homotopy type of the double-directed $n$-cycle and the double-directed $n$-path. It is not hard to see that for any (undirected) graph $G$, the identity $\mathfrak{M}(G)=\Delta(d(G))$ holds, where $d(G)$ is the directed graph on the vertices of $G$ with one edge in each direction between adjacent vertices of $G$. The aforementioned examples studied by Kozlov correspond respectively to the complex of Morse functions of the complete graph, the $n$-cycle and the $n$-path. Complexes of directed graphs have been widely studied (see for example $[3,6,9,10]$ ) and some results of this theory were used in Babson and Kozlov's proof of the Lovász conjecture (see [2]).

In this section we prove Theorem B, which is the special case of Theorem A for regular 1-dimensional CW-complexes. The definition of the complex of Morse functions for regular $\mathrm{CW}$-complexes is identical to the simplicial case. In particular, for a multigraph $G, \mathfrak{M}(G)$ can be viewed as the simplicial complex with one vertex for each directed edge in $G$ and whose simplices are the collections of directed edges which do not form directed cycles.

We first establish the result for simple graphs (i.e. the 1-dimensional case of Theorem A) and then extend it to general multigraphs. We begin by collecting some basic facts about the discrete Morse complex of simple graphs.

Given two simplicial complexes $K, L$, we write $K \equiv L$ if they are isomorphic.

Lemma 3.1. Let $G$ be a connected simple graph. Then,

(1) $\left|V_{\mathfrak{M}(G)}\right|=2\left|E_{G}\right|$.

(2) $\operatorname{dim}(\mathfrak{M}(G))=\left|V_{G}\right|-2$.

Proof. Each edge $e=v w$ gives rise to two primitive vector fields: $(v, e)$ and $(w, e)$. This settles (1). We next prove (2). If $G$ is a tree then it is collapsible and there exists a discrete Morse function $f: G \rightarrow \mathbb{R}$ for which all the edges of $G$ are regular (see [7, Lemma 4.3]). Hence, $\operatorname{dim}(\mathfrak{M}(G))=\left|E_{G}\right|-1=\left|V_{G}\right|-2$. For the general case, proceed by induction on $n=\left|E_{G}\right|$. If $G$ is not a tree, let $V \in \mathfrak{M}(G)$ be of maximal dimension and let $e_{0}, \ldots, e_{r}$ be a cycle in $G$. There must be an edge $e_{i}$ which is critical for $V$ (see [7, Theorem 9.3]). Let $G^{\prime}=G-\left\{e_{i}\right\} . G^{\prime}$ is still connected because $e_{i}$ is in a cycle, $\left|E_{G^{\prime}}\right|=\left|E_{G}\right|-1$ and, by induction, $\operatorname{dim}\left(\mathfrak{M}\left(G^{\prime}\right)\right)=\left|V_{G^{\prime}}\right|-2=\left|V_{G}\right|-2$. Since $V \in \mathfrak{M}\left(G^{\prime}\right)$ and $\operatorname{dim}\left(\mathfrak{M}\left(G^{\prime}\right)\right) \leq \operatorname{dim}(\mathfrak{M}(G))=\operatorname{dim}(V)$, then $\operatorname{dim}(\mathfrak{M}(G))=\left|V_{G}\right|-2$.

Corollary 3.2. If $G, G^{\prime}$ are connected simple graphs such that $\mathfrak{M}(G) \equiv \mathfrak{M}\left(G^{\prime}\right)$ then $\left|V_{G}\right|=\left|V_{G^{\prime}}\right|$ and $\left|E_{G}\right|=\left|E_{G^{\prime}}\right|$. In particular their fundamental groups $\pi_{1}(G)$ and $\pi_{1}\left(G^{\prime}\right)$ are isomorphic.

Remark 3.3. It is easy to check that a vertex $v \in G$ is a leaf if and only if the vertex $(v, e) \in V_{\mathfrak{M}(G)}$ is compatible with every other $\left(u, e^{\prime}\right) \in V_{\mathfrak{M}(G)}$ with the unique exception of $(w, e)$, where $w$ is the other vertex of the edge $e$. This happens if and only if $\operatorname{deg}(v, e)=$ $2\left|E_{G}\right|-2$, where $\operatorname{deg}(v, e)$ is the degree of the vertex $(v, e)$ in the 1-skeleton $\mathfrak{M}(G)^{(1)}$ (i.e. the subcomplex of $\mathfrak{M}(G)$ consisting of the simplices of dimension $\leq 1)$. In particular, if $\mathfrak{M}(G) \equiv \mathfrak{M}\left(G^{\prime}\right)$ then $G$ and $G^{\prime}$ have the same number of leaves.

Let $C_{n}$ denote the simple cycle with $n$ vertices.

Corollary 3.4. Let $G, G^{\prime}$ be two connected simple graphs. If $\mathfrak{M}(G) \equiv \mathfrak{M}\left(G^{\prime}\right)$ and $G=C_{n}$ then $G^{\prime}=C_{n}$.

Proof. By Corollary 3.2, $\left|V_{G}\right|=\left|V_{G^{\prime}}\right|$ and $\left|E_{G}\right|=\left|E_{G^{\prime}}\right|$. Since $G=C_{n}$ then $\left|V_{G}\right|=\left|E_{G}\right|$ and therefore $\left|V_{G^{\prime}}\right|=\left|E_{G^{\prime}}\right|$. Also, since $G$ has no leaves then $G^{\prime}$ has no leaves. Therefore, $G^{\prime}=C_{n}$. 
In order to prove the main results of this paper we will analyze compatibility of regular pairs, similarly to what we did in Remark 3.3. From now on, we write $(\sigma, \tau) \sim(\eta, \rho)$ if $(\sigma, \tau)$ and $(\eta, \rho)$ are compatible, viewed as primitive vector fields (i.e. if they form a simplex in $\mathfrak{M}(K))$, and $(\sigma, \tau) \nsim(\eta, \rho)$ whenever they are not.

Theorem 3.5. Let $G, G^{\prime} \neq C_{n}$ be connected simple graphs and let $F: \mathfrak{M}(G) \rightarrow \mathfrak{M}\left(G^{\prime}\right)$ be a simplicial isomorphism. Define a map $f: G \rightarrow G^{\prime}$ by $f(v)=s(F(v, e))$, where $e$ is any edge incident to $v$ and $s(F(v, e))$ denotes the source simplex of $F(v, e)$. Then $f$ is a well-defined simplicial isomorphism.

Proof. The key part of the proof is to see that $f$ is well-defined, i.e. that $f(v)$ does not depend on the choice of the incident edge $e$. Suppose otherwise and let $\left(v, e_{0}\right),\left(v, e_{1}\right) \in$ $V_{\mathfrak{M}(K)}$ be such that $F\left(v, e_{0}\right)=(w, a)$ and $F\left(v, e_{1}\right)=\left(w^{\prime}, b\right)$ with $w \neq w^{\prime}$. Since $\left(v, e_{0}\right) \nsim$ $\left(v, e_{1}\right)$ then $(w, a) \nsim\left(w^{\prime}, b\right)$ and hence $a=b$ (see Figure 3).
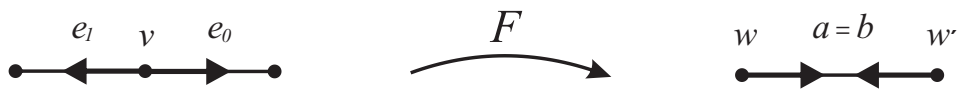

Figure 3

We claim that under this situation we can choose such a vertex $v$ of $G$ with degree greater than or equal to 3 . This will lead to a contradiction since an edge containing $v$ different from $e_{0}$ and $e_{1}$ provides a primitive vector field on $G$ which is incompatible with both $\left(v, e_{0}\right)$ and $\left(v, e_{1}\right)$, while the simplicity of $G^{\prime}$ implies that there is no possible primitive vector field on $G^{\prime}$ incompatible with both $(w, a)$ and $\left(w^{\prime}, a\right)$. To prove this claim, let $e_{1}=v v^{\prime}$ and consider the primitive vector field $\left(v^{\prime}, e_{1}\right)$. Since $\left(w^{\prime}, a\right)=F\left(v, e_{1}\right) \nsim F\left(v^{\prime}, e_{1}\right)$ and $F$ is an isomorphism then there exists an edge $c=w^{\prime} w^{\prime \prime} \in G^{\prime}$ such that $F\left(v^{\prime}, e_{1}\right)=\left(w^{\prime}, c\right)$. Consider now $\left(w^{\prime \prime}, c\right) \in \mathfrak{M}\left(G^{\prime}\right)$. Using a similar argument for $F^{-1}$ and $\left(w^{\prime \prime}, c\right)$ one can find an edge $e_{2} \neq e_{0}, e_{1}$ such that $F^{-1}\left(w^{\prime \prime}, c\right)=\left(v^{\prime}, e_{2}\right)$ (see Figure 4).
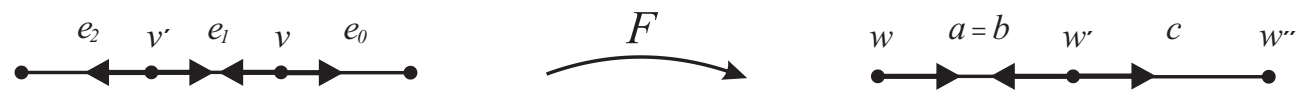

Figure 4

Note that the primitive vector fields $\left(v^{\prime}, e_{1}\right),\left(v^{\prime}, e_{2}\right)$ satisfy the same hypotheses as $\left(v, e_{0}\right),\left(v, e_{1}\right)$ (but replacing $(w, a),\left(w^{\prime}, a\right)$ with $\left(w^{\prime}, c\right),\left(w^{\prime \prime}, c\right)$ respectively). Repeating this argument we obtain a path $e_{1}, e_{2}, e_{3}, \ldots$ where, for any vertex $v \in e_{i} \cap e_{i+1},\left(v, e_{i}\right),\left(v, e_{i+1}\right)$ are mapped to primitive vector fields on $G^{\prime}$ of the form $(u, d),\left(u^{\prime}, d\right)$ with $u \neq u^{\prime}$. By finiteness, this path must form a cycle $C=\left\{e_{j}, e_{j+1}, \ldots, e_{j+k-1}, e_{j+k}=e_{j}\right\}$ for some $j, k$. If $j=0$, since $G$ is not the graph $C_{n}$, by connectedness there is an edge $e \notin C$ intersecting $C$. In this case, $x=e \cap C$ is the desired vertex (see Figure $5(a)$ ). If $j>0$ then the vertex $y=e_{j-1} \cap e_{j}$ is the desired vertex (see Figure $5(b)$ ). This proves that $f$ is well-defined.

We show now that $f$ is a simplicial morphism. Consider an edge $e=v v^{\prime} \in G$. We must see that $f(v) f\left(v^{\prime}\right) \in G^{\prime}$. Since $(v, e) \nsim\left(v^{\prime}, e\right)$ then $F(v, e) \nsim F\left(v^{\prime}, e\right)$. Therefore, either $s(F(v, e))=s\left(F\left(v^{\prime}, e\right)\right)$ or $t(F(v, e))=t\left(F\left(v^{\prime}, e\right)\right)$. In the first case, the same reasoning as above applied to $h=s \circ F^{-1}: G^{\prime} \rightarrow G$ gives a contradiction (replace $v, e_{0}$ and $e_{1}$ with $s(F(v, e))=s\left(F\left(v^{\prime}, e\right)\right), t(F(v, e))$ and $t\left(F\left(v^{\prime}, e\right)\right)$ respectively). Therefore, $t(F(v, e))=t\left(F\left(v^{\prime}, e\right)\right)$ and, in particular, $f(v) f\left(v^{\prime}\right) \in t(F(v, e))$ is an edge in $G^{\prime}$.

Finally, it is easy to see that $f^{-1}=s \circ F^{-1}$ is the inverse of $f$.

Corollary 3.6. Let $G, G^{\prime}$ be connected simple graphs. If $\mathfrak{M}(G) \equiv \mathfrak{M}\left(G^{\prime}\right)$ then $G \equiv G^{\prime}$.

Proof. Follows from Corollary 3.4 and Theorem 3.5. 


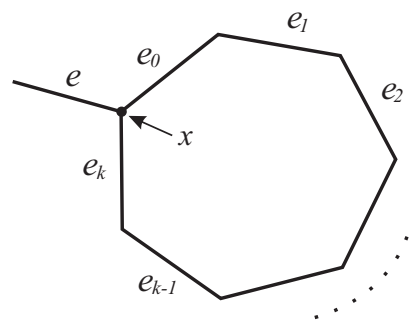

(a)

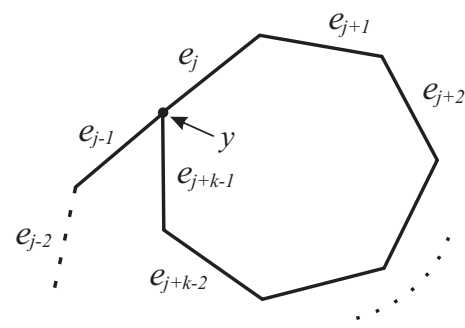

(b)

Figure 5

We now extend the result to multigraphs. Two primitive vector fields $(v, e),\left(v^{\prime}, e^{\prime}\right) \in$ $\mathfrak{M}(G)$ are said to be parallel if $v=v^{\prime}$ and $e$ is parallel to $e^{\prime}$ in $G$. Recall that the link of a simplex $\sigma \in K$ is the subcomplex $l k(\sigma, K)=\{\tau \in K: \tau \cap \sigma=\emptyset, \tau \cup \sigma \in K\}$.

Lemma 3.7. Let $G$ be a connected multigraph with more than two vertices. Then two primitive vector fields $(v, e),\left(v^{\prime}, e^{\prime}\right)$ are parallel in $\mathfrak{M}(G)$ if and only if $(v, e) \nsim\left(v^{\prime}, e^{\prime}\right)$ and $l k((v, e), \mathfrak{M}(G))=l k\left(\left(v^{\prime}, e^{\prime}\right), \mathfrak{M}(G)\right)$.

Proof. Suppose first that $(v, e) \nsim\left(v^{\prime}, e^{\prime}\right)$ and $l k((v, e), \mathfrak{M}(G))=l k\left(\left(v^{\prime}, e^{\prime}\right), \mathfrak{M}(G)\right)$. If $(v, e)$ and $\left(v^{\prime}, e^{\prime}\right)$ are not parallel in $\mathfrak{M}(G)$, then there are only three possibilities for the edges $e$ and $e^{\prime}$ in $G$ which are shown in Figure 6.

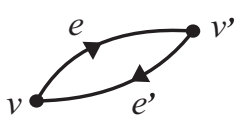

(a)

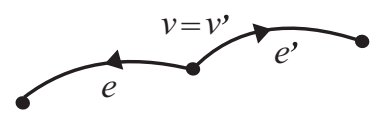

(b)

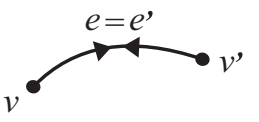

(c)

Figure 6

Since $\left|V_{G}\right| \geq 3$ and $G$ is connected, in each of the three cases, $G$ locally looks as in Figure 7.

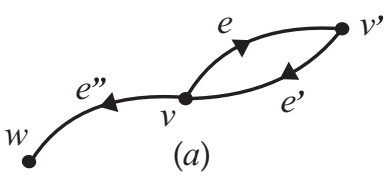

(a)

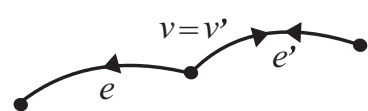

(b)

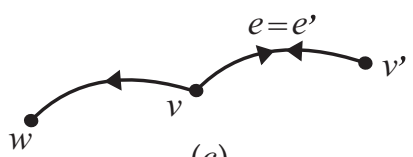

(c)

Figure 7

This contradicts the fact that $l k((v, e), \mathfrak{M}(G))=l k\left(\left(v^{\prime}, e^{\prime}\right), \mathfrak{M}(G)\right)$. The other implication is trivial.

Given a simplicial complex $K$, we define an equivalence relation $\mathcal{R}$ on $V_{K}$ as follows:

$$
v \mathcal{R} w \Leftrightarrow v=w \text { or }\{v, w\} \notin K \text { and } l k(v, K)=l k(w, K) .
$$

Let $\widetilde{K}$ be the simplicial complex whose vertices are the equivalence classes of vertices of $K$ and whose simplices are the sets $\left\{\tilde{v}_{0}, \ldots, \tilde{v}_{r}\right\}$ such that $\left\{v_{0}, \ldots, v_{r}\right\} \in K$. Here $\tilde{v}$ denotes the equivalence class of the vertex $v$. Note that $\widetilde{K}$ is well-defined since, if $v_{i} \mathcal{R} v_{i}^{\prime}$ then $\left\{v_{0}, \ldots, v_{i}, \ldots, v_{r}\right\} \in K$ if and only if $\left\{v_{0}, \ldots, v_{i}^{\prime}, \ldots, v_{r}\right\} \in K$.

Proposition 3.8. Let $K, L$ be simplicial complexes and let $\widetilde{K}$ and $\widetilde{L}$ be as above. If $f: K \rightarrow L$ is a simplicial isomorphism then the map $\tilde{f}: \widetilde{K} \rightarrow \widetilde{L}$ given by $\tilde{f}(\tilde{v})=\widetilde{f(v)}$ is a simplicial isomorphism. 
Proof. We prove first that $\tilde{f}$ is well-defined. Suppose $v \mathcal{R} v^{\prime}$ with $v \neq v^{\prime}$. Since $\left\{v, v^{\prime}\right\} \notin K$ and $f$ is an isomorphism then $\left\{f(v), f\left(v^{\prime}\right)\right\} \notin L$. Also, if $\{f(v)\} \cup \sigma \in L$ then $\{v\} \cup f^{-1}(\sigma) \in$ $K$, which implies that $\left\{v^{\prime}\right\} \cup f^{-1}(\sigma) \in K$. Therefore $\left\{f\left(v^{\prime}\right)\right\} \cup \sigma \in L$.

Finally, $\tilde{f}$ is an isomorphism since $\tilde{f}^{-1}=\widetilde{f^{-1}}$.

Definition. For a multigraph $G$ we define the simplification of $G$, denoted by $s G$, as the simple graph obtained from $G$ by identifying parallel edges.

Remark 3.9. By Lemma 3.7 one can check that the map $f: \widetilde{\mathfrak{M}(G)} \rightarrow \mathfrak{M}(s G)$ defined by $f(\widetilde{(v, e)})=(v, \bar{e})$ is a well-defined isomorphism. Here $\bar{e}$ is the image of the edge $e$ in $s G$.

Proof of Theorem B. Let $F: \mathfrak{M}(G) \rightarrow \mathfrak{M}\left(G^{\prime}\right)$ be an isomorphism. By Proposition 3.8 and Remark 3.9, $F$ induces an isomorphism $\mathfrak{M}(s G) \rightarrow \mathfrak{M}\left(s G^{\prime}\right)$ which we also denote by $F$. By Theorem 3.5 there is an isomorphism $f: s G \rightarrow s G^{\prime}$ sending a vertex $v$ to $s(F(v, e))$ for any edge $e$ incident to $v$. Then, in order to see that $G$ and $G^{\prime}$ are isomorphic, we only need to check that $\left|E_{G}(v, w)\right|=\left|E_{G^{\prime}}(f(v), f(w))\right|$ for any pair of vertices $v, w$ of $G$.

We can suppose that $\left|E_{G}(v, w)\right| \neq 0$ and choose some $e \in E_{G}(v, w)$. Then $(v, e) \in \mathfrak{M}(G)$ and let $e^{\prime}=t(F(v, e)) \in E_{G^{\prime}}(f(v), f(w))$. Note that the set $E_{G}(v, w)$ is in bijection with the set $\{(v, a) \in \mathfrak{M}(G),(v, a) \nsim(w, e)\}$. Similarly, $E_{G^{\prime}}(f(v), f(w))$ is in bijection with $\left\{\left(f(v), a^{\prime}\right) \in \mathfrak{M}\left(G^{\prime}\right),\left(f(v), a^{\prime}\right) \nsim\left(f(w), e^{\prime}\right)\right\}$. By the isomorphism $F$, both sets have the same cardinality.

Chari and Joswig asked in [5] whether there is any connection between the homotopy types of $K$ and $\mathfrak{M}(K)$. They implicitly showed that the homotopy type of $K$ does not determine the homotopy type of $\mathfrak{M}(K)$. For instance, by [5, Proposition 5.1] the complex of Morse functions associated to the 1-simplex is homotopy equivalent to $S^{0}$ and the one associated to the 2-simplex is homotopy equivalent to $S^{1} \vee S^{1} \vee S^{1} \vee S^{1}$. The following example shows that the homotopy type of $\mathfrak{M}(K)$ does not determine the homotopy type of $K$ either.

Example 3.10. Consider the following simple graphs. $G$ has three vertices $u, v, w$ and two edges $u v, u w$. The graph $G^{\prime}$ has four vertices $a, b, c, d$ and four edges $a b, b c, a c, a d$. Note that they are not homotopy equivalent while their associated complexes of Morse functions are both contractible.

\section{Proof of the main Result}

We now extend the result of Corollary 3.6 to simplicial complexes of any dimension. The idea behind the proof is that, in "almost all" cases, a simplicial isomorphism $F$ : $\mathfrak{M}(K) \rightarrow \mathfrak{M}(L)$ restricts to an isomorphism $\left.F\right|_{\mathfrak{M}\left(K^{(1)}\right)}: \mathfrak{M}\left(K^{(1)}\right) \rightarrow \mathfrak{M}\left(L^{(1)}\right)$ between the complexes of discrete Morse functions of the 1-skeleta and by Theorem 3.5 the 1-skeleta of $K$ and $L$ are isomorphic. Then an inductive argument shows that an isomorphism $\mathfrak{M}(K) \equiv \mathfrak{M}(L)$ forces all skeletons of $K$ and $L$ to be isomorphic.

Recall that a cyclic vector field contains at least three regular pairs. One with exactly three regular pairs is said to be minimal and two minimal cyclic vector fields sharing exactly one regular pair are said to be adjacent. Note that a cyclic vector field of index $k$ is equivalent to having an incompatible collection $P=\left\{\left(\sigma_{0}, \tau_{0}\right), \ldots,\left(\sigma_{r}, \tau_{r}\right)\right\}$ of primitive vector fields of index $k \geq 0$ such that every proper subset of $P$ is compatible. Equivalently, the full subcomplex of $\mathfrak{M}(K)$ spanned by the vertices $\left(\sigma_{0}, \tau_{0}\right), \ldots,\left(\sigma_{r}, \tau_{r}\right)$ is the boundary $\partial \Delta^{r}$ of an $r$-simplex.

\section{Remarks 4.1.}


(i) For any pair of complexes $K, L$, a simplicial isomorphism $F: \mathfrak{M}(K) \rightarrow \mathfrak{M}(L)$ sends cyclic vector fields to cyclic vector fields. This follows from the correspondence between cyclic vector fields over a complex $T$ and full subcomplexes of $\mathfrak{M}(T)$ isomorphic to $\partial \Delta^{r}$ (for some $r$ ).

(ii) Note that a simple cycle $e_{0}, \ldots, e_{r}$ in the 1-skeleton of a complex $K$ gives rise to two possible cyclic vector fields of index 0 in $K$ : choosing a vertex $v_{0}$ for $e_{0}$, one of them is $\left\{\left(v_{0}, e_{0}\right),\left(v_{1}, e_{1}\right), \ldots,\left(v_{r}, e_{r}\right)\right\}$ where $v_{i} \neq v_{i+1}$ for all $i=0, \ldots, r-1$. The other cyclic vector field arises from selecting the other vertex of $e_{0}$ to be the source of the primitive vector field.

(iii) It is easy to see that if $\left\{\left(\sigma_{1}, \tau_{1}\right),\left(\sigma_{2}, \tau_{2}\right),\left(\sigma_{3}, \tau_{3}\right)\right\}$ is a minimal cyclic vector field of index $k-1$ then $\left\{\tau_{1}, \tau_{2}, \tau_{3}\right\}$ spans a complex isomorphic to $\Delta^{k-2} * \partial \Delta^{2}$ (here $*$ stands for the join of the complexes). In particular, $\left\langle\tau_{1}, \tau_{2}, \tau_{3}\right\rangle$ is a complex with $k+2$ vertices and a complete 1 -skeleton.

The following result deals with the cases in which an isomorphism $\mathfrak{M}(K) \rightarrow \mathfrak{M}(L)$ does not restrict to an isomorphism $\mathfrak{M}\left(K^{(1)}\right) \rightarrow \mathfrak{M}\left(L^{(1)}\right)$.

Proposition 4.2. Let $K, L$ be connected simplicial complexes and let $F: \mathfrak{M}(K) \rightarrow \mathfrak{M}(L)$ be a simplicial isomorphism. If there exists a primitive vector field $(v, e) \in V_{\mathfrak{M}(K)}$ of index 0 such that $F(v, e)=\left(\sigma^{n-1}, \tau^{n}\right)$ with $n \geq 2$, then $K=L=\partial \Delta^{m}$ for some $m \geq 2$.

Proof. We may assume that $n$ is maximal with the property that there exists $(v, e) \in V_{\mathfrak{M}(K)}$ of index 0 whose image is $\left(\sigma^{n-1}, \tau^{n}\right)$ for some $n \geq 2$. With this assumption, we shall prove that $K=\partial \Delta^{n+1}$. Let $w$ be the other end of $e$. Note that $F(v, e)$ and $F(w, e)$ are incompatible, since $(v, e)$ and $(w, e)$ are incompatible. We conclude that either $s(F(v, e))=$ $s(F(w, e)), s(F(v, e))=t(F(w, e)), t(F(v, e))=s(F(w, e))$ or $t(F(v, e))=t(F(w, e))$. In any case, $\operatorname{since} \operatorname{dim}(s(F(v, e)))>0$, one can find a third primitive vector field over $L$ which is incompatible with both $F(v, e)$ and $F(w, e)$. Since $F$ is an isomorphism, this gives a primitive vector field over $K$ which is incompatible with both $(v, e)$ and $(w, e)$. This forces $e$ to have a proper coface and then $e$ must be a face of a 2 -simplex $\{v, w, u\} \in K$. Let $e^{\prime}=w u$ and $e^{\prime \prime}=u v$ and consider the minimal cyclic vector field $\left\{(v, e),\left(w, e^{\prime}\right),\left(u, e^{\prime \prime}\right)\right\}$ in $K$. By Remark $4.1(i),\left\{F(v, e), F\left(w, e^{\prime}\right), F\left(u, e^{\prime \prime}\right)\right\}$ is a minimal cyclic vector field of index $n-1$ in $L$. Let $F(v, e)=(\sigma, \tau), F\left(w, e^{\prime}\right)=\left(\sigma^{\prime}, \tau^{\prime}\right)$ and $F\left(u, e^{\prime \prime}\right)=\left(\sigma^{\prime \prime}, \tau^{\prime \prime}\right)$. By Remark 4.1 (iii), either $\sigma^{\prime} \prec \tau$ or $\sigma^{\prime} \prec \tau^{\prime \prime}$. We will show that $\sigma^{\prime} \prec \tau$ cannot happen. If this was the case, $F(w, e)=\left(\sigma^{\prime}, \tau\right)$ (being the only regular pair incompatible with both $(\sigma, \tau)$ and $\left(\sigma^{\prime}, \tau^{\prime}\right)$ ). This forces $F\left(u, e^{\prime}\right)=\left(\sigma^{\prime \prime}, \tau^{\prime}\right)$ and $F\left(v, e^{\prime \prime}\right)=\left(\sigma, \tau^{\prime \prime}\right)$ (see Figure 8). Let $\sigma^{\prime \prime \prime} \prec \tau$ be different from $\sigma, \sigma^{\prime}$. Consider a minimal cyclic vector field of index $n-2\left\{(\rho, \sigma),\left(\rho^{\prime}, \sigma^{\prime}\right),\left(\rho^{\prime \prime \prime}, \sigma^{\prime \prime \prime}\right)\right\}$ in the boundary of $\tau$. Now, the source of $F^{-1}(\rho, \sigma)$ must be the vertex $v$ since this is the only way $F^{-1}(\rho, \sigma)$ is incompatible with both $F^{-1}(\sigma, \tau)=(v, e)$ and $F^{-1}\left(\sigma, \tau^{\prime \prime}\right)=\left(v, e^{\prime \prime}\right)$. A similar reasoning shows that $s\left(F^{-1}\left(\rho^{\prime}, \sigma^{\prime}\right)\right)=w$ and $s\left(F^{-1}\left(\rho^{\prime \prime \prime}, \sigma^{\prime \prime \prime}\right)\right)=u$. Therefore, the preimage by $F$ of the cyclic vector field $\left\{(\rho, \sigma),\left(\rho^{\prime}, \sigma^{\prime}\right),\left(\rho^{\prime \prime \prime}, \sigma^{\prime \prime \prime}\right)\right\}$ does not constitute a cyclic vector field in $K$ (see Figure 8), which contradicts the fact that $F$ is an isomorphism.

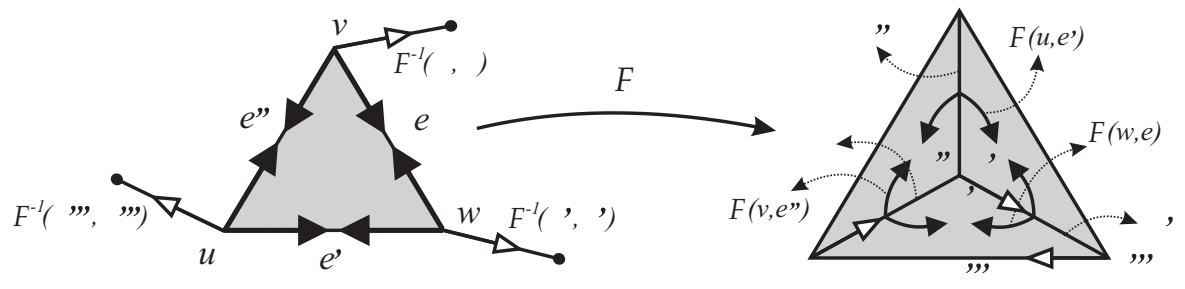

Figure 8. The image of $\left(v, e^{\prime \prime}\right),\left(u, e^{\prime}\right)$ and $(w, e)$ in the case $\sigma^{\prime} \prec \tau$. The minimal cyclic vector field of index $n-2\left\{(\rho, \sigma),\left(\rho^{\prime}, \sigma^{\prime}\right),\left(\rho^{\prime \prime \prime}, \sigma^{\prime \prime \prime}\right)\right\}$ appears in white arrows. 
Therefore, we must have $\sigma^{\prime} \prec \tau^{\prime \prime}$ and a similar reasoning as before shows that the situation must be as in Figure 9 . Let $Q$ be the subcomplex of $L$ generated by the $n$-simplices $\tau, \tau^{\prime}, \tau^{\prime \prime}$ and note that $Q$ has $n+2 \geq 4$ vertices and a complete 1-skeleton (see Remark 4.1 (iii)). Let $S$ denote the collection of all primitive vector fields in $Q$ of index 0 and let $G(x, a)=t\left(F^{-1}(x, a)\right) \in K$ for each $(x, a) \in S$. We will prove that $K=\partial \Delta^{n+1}$ in various steps.

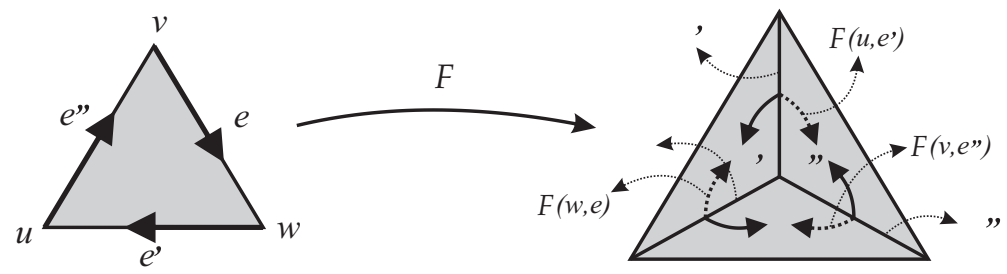

Figure 9

STEP 1. We show first that $G(S)$ is a collection of $k$-simplices for a fixed $k \leq n$. Consider a sequence $\tau=\eta^{n} \succ \sigma=\eta^{n-1} \succ \eta^{n-2} \succ \cdots \succ \eta^{1} \succ \eta^{0}=y$ of faces of consecutive decreasing dimension of the $n$-simplex $\tau$ ending in a vertex $y$ of $\tau$. Each pair $\left(\eta^{i-1}, \eta^{i}\right)$ is incompatible with the previous and the next pair. Since incompatibility between two regular pairs arises if they share the same source, the same target or the source of one is the target of the other, then the biggest difference in dimension between $F^{-1}\left(\eta^{0}, \eta^{1}\right)=F^{-1}\left(y, \eta^{1}\right)$ and $F^{-1}\left(\eta^{n-1}, \eta^{n}\right)=F^{-1}(\sigma, \tau)$ occurs if the target of $\left(\eta^{i-1}, \eta^{i}\right)$ is the source of $\left(\eta^{i}, \eta^{i+1}\right)$ for all $i=0, \ldots, n-1$. We conclude that $F^{-1}\left(y, \eta^{1}\right)=\left(\psi^{k-1}, \rho^{k}\right)$ for some $k \leq n$. Now, since $Q$ has a complete 1-skeleton then any edge $a \in Q$ is part of a cycle also containing $\eta^{1}$. Therefore, any $(x, a) \in S$ is part of a cyclic vector field of index 0 containing either $\left(y, \eta^{1}\right)$ or $\left(z, \eta^{1}\right)$, where $z$ is the other end of $\eta^{1}$ (see Remark $4.1(i i))$. Since $F$ maps cyclic vector fields to cyclic vector fields, it suffices to show that $t\left(F^{-1}\left(z, \eta^{1}\right)\right)$ is also a $k$-simplex. But since $\left|V_{Q}\right| \geq 4$, we can form a cyclic vector field of index 0 containing $\left(y, \eta^{1}\right)$ and a new pair $(p, \psi)$, and another one containing $\left(z, \eta^{1}\right)$ and $(p, \psi)$ as shown in Figure 10.
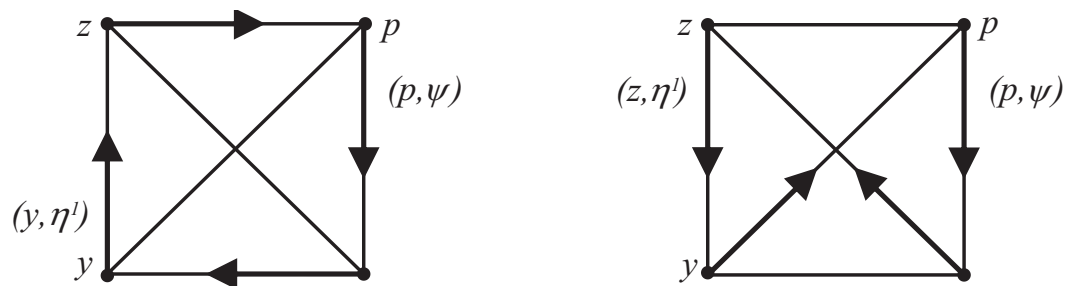

Figure 10

SteP 2. We show that $k=n$ and that $G(S)$ spans $\partial \Delta^{n+1}$. Fix a minimal cyclic vector field $C_{1}=\left\{\left(v_{1}, v_{1} v_{2}\right),\left(v_{2}, v_{2} v_{3}\right),\left(v_{3}, v_{1} v_{3}\right)\right\}$ in $Q$ and let $T$ be the subcomplex of $K$ generated by the three $k$-simplices in $G\left(C_{1}\right)$. Note that $\left|V_{T}\right|=k+2$ by Remark 4.1 (iii). We claim that all $k$-simplices in $G(S)$ have their vertices in $V_{T}$. To see this, let $(x, a) \in S$ and let $y$ be the other end of $a$. All possible situations for $(x, a)$ with respect to $C_{1}$ are considered in Figure 11 where one can verify that it is always possible to find a sequence of adjacent minimal cyclic vector fields between $C_{1}$ and a minimal cyclic vector field containing $(x, a)$. By an inductive argument it suffices to show that the image by $G$ of a regular pair in a minimal cyclic vector field adjacent to $C_{1}$ has its vertices in $V_{T}$. Let $C_{2}=\left\{\left(v_{2}, v_{2} v_{3}\right),\left(v_{3}, v_{3} v_{4}\right),\left(v_{4}, v_{2} v_{4}\right)\right\}$ be one such cyclic vector field. Since the 


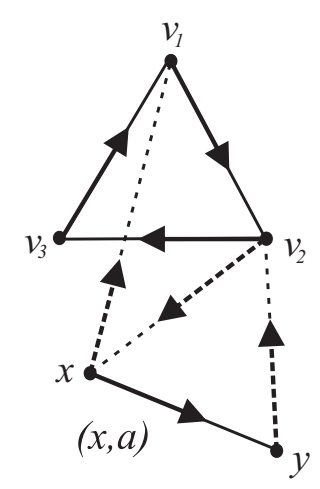

(a) (b)

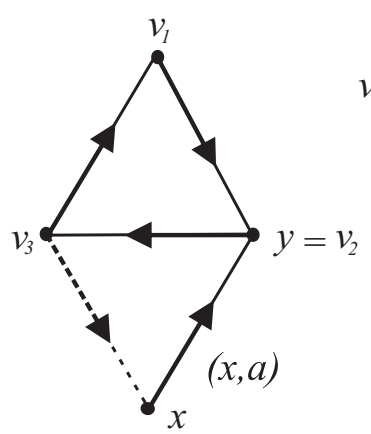

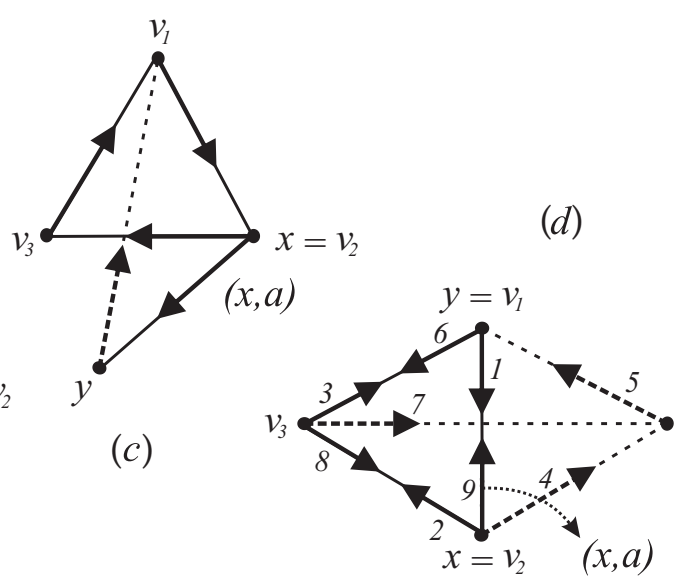

Figure 11. All possible configurations of the relation between $(x, a)$ and $C_{1}$ are obtained by permutation of $\left\{v_{1}, v_{2}, v_{3}\right\}$. Note that the sequence of adjacent minimal cyclic vector fields in situation $(d)$ is given by $C_{1}=\{1,2,3\},\{1,4,5\},\{5,6,7\}$ and $\{6,8,9=(x, a)\}$.

$k$-simplex $G\left(v_{2}, v_{2} v_{3}\right) \in G\left(C_{1}\right) \cap G\left(C_{2}\right)$, by Remark 4.1 (iii) it suffices to show that the only vertex $q \in V_{T} \backslash V_{G\left(v_{2}, v_{2} v_{3}\right)}$ is also in $G\left(C_{2}\right)$. But since $\left(v_{3}, v_{3} v_{4}\right) \nsim\left(v_{3}, v_{1} v_{3}\right)$ then either $s\left(F^{-1}\left(v_{3}, v_{3} v_{4}\right)\right)=s\left(F^{-1}\left(v_{3}, v_{1} v_{3}\right)\right)$ or $t\left(F^{-1}\left(v_{3}, v_{3} v_{4}\right)\right)=t\left(F^{-1}\left(v_{3}, v_{1} v_{3}\right)\right)$. Since the configuration must be as shown in Figure 9 then the only two possible cases are shown in Figure 12. This proves that $q \in G\left(C_{2}\right)$.
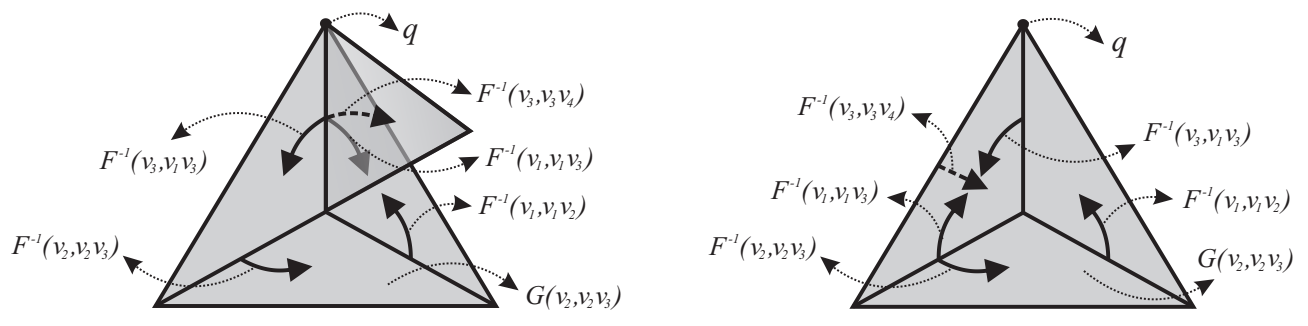

Figure 12. Here $F^{-1}\left(v_{3}, v_{3} v_{4}\right)$ is drawn with a dashed arrow. On the left: the case $s\left(F^{-1}\left(v_{3}, v_{3} v_{4}\right)\right)=s\left(F^{-1}\left(v_{3}, v_{1} v_{3}\right)\right)$ cannot happen because the subcomplex of $G\left(C_{2}\right)$ spanned by the two $k$-simplices $G\left(v_{2}, v_{2} v_{3}\right)$ and $t\left(F^{-1}\left(v_{3}, v_{3} v_{4}\right)\right)$ has more than $k+2$ vertices, which contradicts Remark 4.1 (iii) for $C_{2}$. On the right: in the case $t\left(F^{-1}\left(v_{3}, v_{3} v_{4}\right)\right)=t\left(F^{-1}\left(v_{3}, v_{1} v_{3}\right)\right)$ we readily see that $q \in G\left(C_{2}\right)$.

Now, since $Q$ has a complete 1-skeleton then we can form a cycle in $Q^{(1)}$ containing all the vertices of $Q$. The corresponding cyclic vector field of index 0 has as a preimage by $F$ a cyclic vector field of index $k-1$ with $n+2$ regular pairs. By definition of cyclic vector field, the target of all these pairs are distinct $k$-simplices. Therefore, we conclude that $k=n$ and that $G(S)$ spans $\partial \Delta^{n+1}$.

STEP 3. We show that $K$ is spanned by $G(S)$. Let $\langle G(S)\rangle$ denote the subcomplex of $K$ spanned by $G(S)$. First, note that two primitive vector fields $(x, a),(x, b)$ of index 0 over $\langle G(S)\rangle$ sharing the same source vertex $x$ are mapped by $F$ to primitive vector fields with the same target $n$-simplex. This follows from the reasoning made at the beginning of the proof (see Figure 9, setting $v=x, e=a$ and $e^{\prime \prime}=b$ ). Since $F$ is an isomorphism we conclude that, for an $n$-simplex $\rho$ in the image of $\langle G(S)\rangle$, the $n+1$ primitive vector fields with target $\rho$ are precisely the image of the $n+1$ primitive vector fields of index 0 over $\langle G(S)\rangle=\partial \Delta^{n+1}$ sharing a same source vertex. 
Suppose that $K-\langle G(S)\rangle \neq \emptyset$. By connectivity, there is an edge $\tilde{e} \in K-\langle G(S)\rangle$ such that $\tilde{e} \cap\langle G(S)\rangle$ contains a vertex $z$. Let $\tilde{e}^{\prime}, \tilde{e}^{\prime \prime} \in\langle G(S)\rangle=\partial \Delta^{n+1}$ with $\tilde{e}^{\prime} \neq \tilde{e}^{\prime \prime}$ and consider the primitive vector fields $(z, \tilde{e}),\left(z, \tilde{e}^{\prime}\right),\left(z, \tilde{e}^{\prime \prime}\right)$. Since $(z, \tilde{e}) \nsim\left(z, \tilde{e}^{\prime}\right)$ and $(z, \tilde{e}) \nsim\left(z, \tilde{e}^{\prime \prime}\right)$ then $F(z, \tilde{e}) \nsim F\left(z, \tilde{e}^{\prime}\right)$ and $F(z, \tilde{e}) \nsim F\left(z, \tilde{e}^{\prime \prime}\right)$. Since $n$ was taken to be maximal with the property that there exists $(v, e) \in V_{\mathfrak{M}(K)}$ of index 0 whose image is $\left(\sigma^{n-1}, \tau^{n}\right)$, we conclude that $t(F(z, \tilde{e}))$ must be equal to $t\left(F\left(z, \tilde{e}^{\prime}\right)\right)=t\left(F\left(z, \tilde{e}^{\prime \prime}\right)\right)$ for the above incompatibilities to hold. By the previous remarks, this is a contradiction since all $n+1$ primitive vector fields whose target is this $n$-simplex are in the image of the $n+1$ primitive vector fields in $S$ with source $z$. This concludes the proof.

Similarly as we did with the edges of simple graphs, for simplicity of notation, an $n$-simplex $\sigma=\left\{v_{0}, \ldots, v_{n}\right\} \in K$ will be denoted by $\sigma=v_{0} \cdots v_{n}$.

Proof of Theorem A. Let $F: \mathfrak{M}(K) \rightarrow \mathfrak{M}(L)$ be an isomorphism. By Proposition 4.2 we may assume that every primitive vector field of index 0 in $\mathfrak{M}(K)$ (resp. in $\mathfrak{M}(L)$ ) is mapped by $F$ (resp. by $F^{-1}$ ) to a primitive vector field of index 0 . This gives a welldefined isomorphism $\left.F\right|_{\mathfrak{M}\left(K^{(1)}\right)}: \mathfrak{M}\left(K^{(1)}\right) \rightarrow \mathfrak{M}\left(L^{(1)}\right)$. By Theorem 3.5 there exists an isomorphism $f: K^{(1)} \rightarrow L^{(1)}$ with $f(v)=s(F(v, e))$ for any $e \succ v$. Note that for every edge $x y \in K$ we have $F(x, x y)=(f(x), f(x) f(y))$. We will show by induction that for any $(n+1)$-simplex $v_{0} \cdots v_{n+1}$,

$$
F\left(v_{0} \cdots v_{n}, v_{0} \cdots v_{n} v_{n+1}\right)=\left(f\left(v_{0}\right) \cdots f\left(v_{n}\right), f\left(v_{0}\right) \cdots f\left(v_{n}\right) f\left(v_{n+1}\right)\right) .
$$

Given $\tau=v_{0} \cdots v_{n+1} \in K$, consider the following two families of primitive vector fields:

- $I=\left\{\left(v_{0} \cdots \hat{v}_{i} \cdots v_{n}, v_{0} \cdots v_{n}\right), 0 \leq i \leq n\right\}$

- $J=\left\{\left(v_{1} \cdots \hat{v}_{j} \cdots v_{n+1}, v_{1} \cdots v_{n+1}\right), 1 \leq j \leq n+1\right\}$

where the hat over a vertex means that that vertex is to be omitted. By induction,

- $F\left(v_{0} \cdots \hat{v}_{i} \cdots v_{n}, v_{0} \cdots v_{n}\right)=\left(f\left(v_{0}\right) \cdots \widehat{f\left(v_{i}\right)} \cdots f\left(v_{n}\right), f\left(v_{0}\right) \cdots f\left(v_{n}\right)\right)$ and

- $F\left(v_{1} \cdots \hat{v}_{j} \cdots v_{n+1}, v_{1} \cdots v_{n+1}\right)=\left(f\left(v_{1}\right) \cdots \widehat{f\left(v_{j}\right)} \cdots f\left(v_{n+1}\right), f\left(v_{1}\right) \cdots f\left(v_{n+1}\right)\right)$.

Since $\left(v_{0} \cdots v_{n}, v_{0} \cdots v_{n+1}\right) \in \mathfrak{M}(K)$ is incompatible with every element of $I$ then the only possibility is that the source of $F\left(v_{0} \cdots v_{n}, v_{0} \cdots v_{n+1}\right)$ is $f\left(v_{0}\right) \cdots f\left(v_{n}\right)$; that is,

$$
F\left(v_{0} \cdots v_{n}, v_{0} \cdots v_{n+1}\right)=\left(f\left(v_{0}\right) \cdots f\left(v_{n}\right), f\left(v_{0}\right) \cdots f\left(v_{n}\right) w\right)
$$

for some vertex $w \in L$. Analogously, since $\left(v_{1} \cdots v_{n+1}, v_{0} \cdots v_{n+1}\right) \in \mathfrak{M}(K)$ is incompatible with every element of $J$ then

$$
F\left(v_{1} \cdots v_{n+1}, v_{0} \cdots v_{n+1}\right)=\left(f\left(v_{1}\right) \cdots f\left(v_{n+1}\right), f\left(v_{1}\right) \cdots f\left(v_{n+1}\right) u\right)
$$

for some vertex $u \in L$. But $\left(v_{0} \cdots v_{n}, v_{0} \cdots v_{n+1}\right) \nsim\left(v_{1} \cdots v_{n+1}, v_{0} \cdots v_{n+1}\right)$, so we must have $f\left(v_{0}\right) \cdots f\left(v_{n}\right) w=f\left(v_{1}\right) \cdots f\left(v_{n+1}\right) u$, and therefore $w=f\left(v_{n+1}\right)$ and $u=f\left(v_{0}\right)$.

\section{REFERENCES}

[1] R. Ayala, L. M. Fernández, A. Quintero, and J. A. Vilches. A note on the pure Morse complex of a graph. Topology Appl. 155 (2008), No. 17-18, 2084-2089.

[2] E. Babson, D. N. Kozlov. Proof of the Lovász conjecture. Ann. of Math. (2) 165 (2007), No. 3, 965-1007.

[3] A. Björner, V. Welker. Complexes of directed graphs. SIAM J. Discrete Math. 12 (1999), No. 4, 413-424 (electronic).

[4] M. K. Chari. On discrete Morse functions and combinatorial decompositions. Discrete Math. 217 (2000), No. 1-3, 101-113.

[5] M. K. Chari, M. Joswig. Complexes of discrete Morse functions. Discrete Math. 302 (2005), No. 1-3, 3951.

[6] A. Engström. Complexes of directed trees and independence complexes. Discrete Math. 309 (2009), No. 10, 3299-3309.

[7] R. Forman. Morse theory for cell complexes. Adv. Math. 134 (1998), No. 1, 90-145.

[8] R. Forman. Witten-Morse theory for cell complexes. Topology 37 (1998), No. 5, 945-979. 
[9] D. Jojić. Shellability of complexes of directed trees. Filomat 27 (2013), No. 8, 1551-1559.

[10] D. N. Kozlov. Complexes of directed trees. J. Combin. Theory Ser. A 88 (1999), No. 1, 112-122.

[11] A. Lundell, S. Weingram. The topology of CW complexes. Van Nostrand - Reinhold, New York (1969).

Departamento de Matemática-Imas, FCEyn, Universidad de Buenos Aires, Buenos Aires, Argentina.

E-mail address: ncapitel@dm.uba.ar

E-mail address: gminian@dm.uba.ar 